\title{
El desinterés en recibir la vacuna contra el coronavirus en población de 23 ciudades colombianas, julio a diciembre de $2020^{*}$
}

\section{Lack of interest in receiving the coronavirus vaccine in the population of 23 Colombian cities, July-December 2020}

\section{0 esinteresse em receber a vacina contra coronavírus na população de 23 cidades colombianas, julho a dezembro de 2020}

Recibido: 25 de Febrero de 2021. Aceptado: 06 de Abril de

2021. Publicado: 30 de diciembre de 2021.

DOI: https://doi.org/10.11144/Javeriana.rgps20.drvc

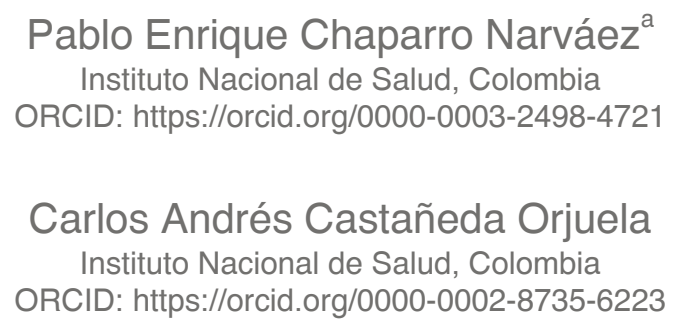

Para citar este artículo Chaparro PE, Castañeda CA. El desinterés en recibir la vacuna contra el coronavirus en población de 23 ciudades colombianas, julio a diciembre de 2020. Rev Gerenc Polit Salud. 2021;20. https://doi. org/10.11144/Javeriana.rgps20.drvc

\footnotetext{
* Artículo de investigación

${ }^{\text {a }}$ Autor de correspondencia. Correo electrónico: pchaparro@ins.gov.co
} 


\section{Resumen}

Introducción. La falta de interés de la población en ser vacunada contra el SARS-CoV-2 representa un problema para el manejo de la pandemia. Objetivo. El objetivo fue estimar la falta de interés en recibir la vacuna contra el coronavirus en población de 23 ciudades colombianas, entre julio y diciembre de 2020. Métodos. Se diseñó un estudio descriptivo que empleó información de la Encuesta Pulso Social del DANE en la que se indagó a la población sobre la intención en aplicarse la vacuna y las razones para rechazarla. Se estimaron la prevalencia del desinterés en recibir la vacuna y las diferencias entre las estimaciones de diciembre y julio. Resultados. La prevalencia fue más alta en mujeres, mayores de 54 años, personas sin ninguna escolaridad, hogares con dos personas o menos, grupos pobres y en ciudades como Cali y Bucaramanga. Las diferencias entre diciembre y julio señalaron aumento del desinterés en menores de 25 años y en Santa Marta y Cartagena; el comportamiento del periodo fue ascendente. La principal razón para no querer recibir la vacuna fue la creencia de que esta puede ser insegura debido a los potenciales efectos adversos. Se identificaron variaciones demográficas y geográficas en las prevalencias de no aceptación. Conclusión. Esta investigación puede ayudar a las autoridades de salud a elaborar políticas relacionadas con las estrategias educativas y de comunicación destinadas a aumentar la confianza en la eficacia y seguridad de la vacuna.

Palabras clave: Vacunación, intención, negativa a la vacunación, enfermedades prevenibles por vacunación, COVID-19, Colombia.

\section{Abstract}

Introduction. The population's lack of interest in being vaccinated against coronavirus is a problem for pandemic management. Objective. To estimates the lack of interest in receiving the coronavirus vaccine in the population of 23 Colombian cities between July and December 2020. Methods. A descriptive study using information from the Social Pulse Survey - DANE- was done. The Social Pulse Survey asked participants about their interest to get the vaccine and the reasons for refusing it. The Prevalence of interest in not receiving the vaccine and differences between December to July were estimated. Results. The prevalence was highest in women, people over 54 years of age, population without schooling, households with two people or less, poor groups, and cities like Cali and Bucaramanga. Differences between December and July showed a further increase in people under 25 years of Santa Marta and Cartagena. The main reason for refusing the vaccine was the belief that this may be unsafe due to potential side effects. There were geographic and demographic variations in the prevalence of non-acceptance. Conclusions. This research can help health authorities to develop policies related to education and communication strategies that could increase the confidence in the efficacy and safety of the vaccine.

Keywords: Vaccination, intention, vaccination refusal, vaccine-preventable diseases, COVID-19, Colombia.

\section{Resumo}

Introdução. O desinteresse da população em se vacinar contra a SARS-CoV-2 representa um problema para o manejo da pandemia. Objetivo. O objetivo foi estimar o desinteresse em receber a vacina contra o coronavírus na população de 23 cidades colombianas entre julho e dezembro de 2020. Métodos. Foi elaborado um estudo descritivo que utilizou informações da Pesquisa Pulso Social do DANE, a qual indagou sobre o interesse em tomar a vacina e os motivos para rejeitá-la. Foram estimadas as prevalências do interesse em não receber a vacina e as diferenças entre as estimativas para dezembro e julho. Foram identificadas variações demográficas e geográficas nas prevalências de não aceitação. Resultados. A prevalência foi maior em mulheres, com mais de 54 anos, sem escolaridade, domicílios com duas pessoas ou menos, grupos pobres e em cidades como Cali e Bucaramanga. As diferenças entre dezembro e julho indicaram um aumento do desinteresse em menores de 25 anos e em Santa Marta e Cartagena. O principal motivo do não interesse em receber a vacina é a crença de que ela pode ser insegura devido a potenciais efeitos adversos. Conclusão. Esta pesquisa pode ajudar as autoridades de saúde a desenvolver políticas relacionadas às estratégias educacionais e de comunicação destinadas a aumentar a confiança na eficácia e segurança da vacina.

Palavras-chave: Vacinação, intenção, recusa de vacinação, doenças evitáveis por vacina, COVID-19, Colômbia. 
El desinterés en recibir la vacuna contra el coronavirus en

población de 23 ciudades colombianas, julio a diciembre de 2020

\section{Introducción}

A finales de febrero de 2021 el SARS-CoV-2 había infectado a más de 111 millones de personas y ocasionado alrededor de 2,5 millones de muertes en todo el mundo, incluidas las 60.000 defunciones ocurridas en Colombia (1). Luego de más de un año desde su aparición en el que se ha recurrido a diversas medidas para su control y prevención, finalmente se desarrollaron las vacunas y se autorizó su uso de emergencia. La vacunación se ha identificado como un aspecto clave para la prevención y eliminación de la enfermedad (2) y su éxito dependerá de la eficacia, aceptación y cobertura que se alcance $(3,4)$.

Las encuestas y los estudios que se realizaron durante el año 2020 indagando sobre la intención de la población para ser vacunada revelaron diferencias en las distintas áreas geográficas. Los resultados indicaron que, en junio de 2020, en Estados Unidos el 20\% de las personas no se vacunarían (5), luego entre octubre y diciembre las cifras de los que no lo harían variaron entre $27 \%$ y $60 \%$ (6-9). En Francia, en marzo y en abril, una cuarta parte de los participantes afirmó que rechazaría la vacuna $(10,11)$. En el estudio realizado en abril que incluyó habitantes de Dinamarca, Francia, Alemania, Italia, Portugal, los Países Bajos y el Reino Unido, señaló que el 7,2\% de los participantes no deseaba vacunarse (12). Otro estudio realizado en junio que incluyó individuos de 19 países informó que el 14,2\% se rehusaría a recibir la vacuna (13). En toda Europa, en agosto, el 26\% de las personas no planeaba vacunarse (14). Para noviembre, en España, se informaba que el $47 \%$ de habitantes tampoco accedería a vacunarse (15).

El retraso en la aceptación de la vacunación, a pesar de la disponibilidad de servicios de vacunación, es un problema que se viene reconociendo aun antes de la actual emergencia en salud pública por COVID-19. En 2019, la Organización Mundial de la Salud (OMS) lo había incluido entre los diez problemas de salud que abordaría (16). Esta situación se presenta sobre todo con las vacunas nuevas o recién introducidas; varía entre y dentro de los países, con el contexto, el tiempo, el lugar, el programa y la vacuna específica y está influenciada por la complacencia, la conveniencia y la confianza (17).

En la explicación de la falta de interés por recibir una vacuna se han utilizado los modelos tradicionales de promoción de la salud, entre ellos el de creencias sobre la salud (18) Sin embargo, en los últimos años, el modelo de rechazo científico de las "raíces de la actitud" ha identificado valores como las creencias conspirativas, la reactancia, la sensibilidad al disgusto y las visiones del mundo individuales/jerárquicas asociadas con las actitudes contra la vacunación (19). Desde el punto de vista de la prevención, entre los factores que afectan la aceptación de las vacunas se incluyen la falta percibida de una recomendación clara $(20,21)$, el riesgo subjetivo de la población (incluidos los factores de peligro e indignación) (22,23), las redes sociales (24) y la creencia en la seguridad de la vacuna (25). Asimismo, factores asociados con la renuencia a aceptar una vacuna, incluida la falta de atención a factores de indignación, preocupaciones sobre la seguridad de las vacunas, desconfianza y temores sobre las motivaciones para la vacunación (23). 
La falta de intención en recibir la vacuna representa un peligro tanto para el individuo como para su comunidad, ya que la exposición a una enfermedad infecciosa pone a la persona en riesgo, y es más probable que los individuos transmitan la enfermedad a otros si no se vacunan (26). En el caso de la COVID-19, la perspectiva a corto y mediano plazo es que solo la aplicación de una vacuna efectiva a escala masiva será capaz de controlar el nivel de transmisión, por lo que, si una importante proporción de la población a riesgo de infección se niega a aplicarse la vacuna, el control de la pandemia estará más lejano (27). El objetivo de este análisis fue describir el no tener interés en recibir la vacuna contra el coronavirus en la población de 23 ciudades colombianas, entre julio y diciembre de 2020.

\section{Material y métodos}

\section{Tipo de estudio}

Se llevó a cabo un estudio descriptivo para determinar la falta de interés en recibir la vacuna contra el SARS-CoV-2 en Colombia, entre julio y diciembre de 2020.

\section{Fuente de información}

Los datos se obtuvieron de la Encuesta Pulso Social realizada por el Departamento Administrativo Nacional de Estadística (DANE), que surgió de la necesidad de contar con información sobre el impacto de la situación generada por el COVID-19 en la población colombiana (28). La Encuesta Pulso Social inició en julio de 2020 y mensualmente recopiló información hasta completar seis rondas. Esta encuesta fue una submuestra de la Gran Encuesta Integrada de Hogares (GEIH). El universo de la GEIH estuvo conformado por población civil no institucionalizada, residente en todo el territorio nacional, y utilizó muestreo probabilístico, multietápico, estratificado, de conglomerados desiguales y autoponderado $(29,30)$.

La Encuesta Pulso Social cubrió 23 ciudades capitales de departamento y áreas metropolitanas (AM): Barranquilla, Cartagena, Sincelejo, Valledupar, Santa Marta, Riohacha, Bogotá, Tunja, Villavicencio, Neiva, Florencia, Bucaramanga, Cúcuta, Cali, Pasto, Popayán, Manizales, Ibagué, Pereira, Armenia, Medellín, Montería, Quibdó. Por cada hogar seleccionó una persona -el jefe del hogar o su cónyuge-, recopiló la información a través de entrevista telefónica y la registró en un formulario web.

La Encuesta Pulso Social constaba de 56 preguntas agrupadas en cinco dimensiones: confianza del consumidor, bienestar subjetivo, redes de apoyo de los hogares, bienestar de los hogares que cuentan con niños, niñas y adolescentes y conocimiento y acceso a las políticas nacionales y locales de apoyo a los hogares. Para efectos de este estudio se consideró la dimensión de bienestar subjetivo y específicamente las preguntas "En caso de que se encontrara disponible, ¿estaría usted interesado(a) en aplicarse la vacuna en contra del coronavirus?” y “¿Cuál es la 
razón principal por la que no está interesado/a en aplicarse la vacuna en contra del coronavirus?". En la tabla 1 se presenta el tamaño de la muestra por cada ronda.

Tabla 1 Rondas, muestra y periodo de recolección de la información, Encuesta Pulso Social, Colombia, 2020

\begin{tabular}{|c|c|c|c|}
\hline \multirow{2}{*}{$\begin{array}{l}\text { Periodo de } \\
\text { referencia }\end{array}$} & Muestra & $\begin{array}{c}\text { Muestra } \\
\text { expandida }\end{array}$ & \multirow[t]{2}{*}{ Periodo de recolección } \\
\hline & n & $\mathrm{n}$ & \\
\hline Julio & 9.866 & 11.894 .323 & 13 de julio a 9 de agosto de 2020 \\
\hline Agosto & 10.588 & 12.174 .770 & 10 de agosto a 6 de septiembre de 2020 \\
\hline Septiembre & 10.909 & 12.015 .589 & 7 de septiembre a 4 de octubre de 2020 \\
\hline Octubre & 11.580 & 12.065 .548 & 5 de octubre a 8 de noviembre de 2020 \\
\hline Noviembre & 11.073 & 12.028 .762 & 9 de noviembre a 6 de diciembre de 2020 \\
\hline Diciembre & 10.602 & 12.099 .790 & 7 de diciembre de 2020 a 3 de enero de 2021 \\
\hline
\end{tabular}

Fuente: elaboración propia con base en la Encuesta Pulso Social, 2020, DANE.

\section{Variables de estudio}

Para la pregunta "En caso de que se encontrara disponible, ¿estaría usted interesado(a) en aplicarse la vacuna en contra del coronavirus?", las variables consideradas de julio a diciembre fueron mes, número de personas entrevistadas (muestra expandida), sexo, grupo de edad, nivel educativo y tamaño del hogar; y de septiembre a diciembre pobreza monetaria y ciudad.

Para la pregunta “¿Cuál es la razón principal por la que no está interesado/a en aplicarse la vacuna en contra del coronavirus?", la información se recolectó a partir de noviembre y contempló las opciones de "no cree que la vacuna pueda ser lo suficientemente efectiva", "cree que la vacuna puede ser insegura debido a los potenciales efectos adversos", "no cree que el coronavirus sea peligroso para su salud", "está en contra de las vacunas en general", "cree que la vacuna se va a utilizar para manipularlo", "ya estuvo contagiado del coronavirus y se recuperó" y "otra".

\section{Análisis estadístico}

Para las variables cualitativas se calcularon frecuencias y porcentajes. Se estimó la prevalencia del desinterés en recibir la vacuna contra el coronavirus. Se calcularon las diferencias entre las estimaciones de diciembre y julio (de diciembre y septiembre y de diciembre y noviembre de acuerdo con la información recolectada) con sus respectivos intervalos de confianza del 95\%, considerando una prueba de ji cuadrado. Para examinar el comportamiento del desinterés en recibir la vacuna se emplearon los porcentajes obtenidos en cada ronda para cada una de las variables consideradas. Para el procesamiento de la información se emplearon hojas de cálculo de Microsoft Excel ${ }^{\mathrm{TM}}$. La información se analizó con el lenguaje de programación R. 


\section{Resultados}

Las características de quienes respondieron la encuesta en julio y en diciembre fueron muy similares. La mayoría de participantes está compuesta por mujeres $(62,8 \%$ y $61,8 \%)$ que tenían entre 25 y 54 años (58,4\% y 59,7\%), alcanzaron una escolaridad de nivel medio (31,2\% y $34,2 \%$ ), provenían de hogares conformados por 4 o más personas $(45,5 \%$ y $44,5 \%)$, pertenecían al grupo de no pobres $(77,7 \%$ y 78,4\%) y principalmente vivían en Bogotá $(34,6 \%$ y $35,0 \%)$ y Medellín y su AM (15,6\% y 15,7\%). Ver la tabla 2.

Tabla 2 Características de los participantes en la Encuesta Pulso Social, Colombia, julio - diciembre 2020

\begin{tabular}{|c|c|c|c|c|}
\hline \multirow[t]{2}{*}{ Categorías } & \multicolumn{2}{|c|}{$\begin{array}{c}\text { Julio } \\
(\mathbf{n}=\mathbf{1 1 . 8 9 4 . 3 2 3})\end{array}$} & \multicolumn{2}{|c|}{$\begin{array}{c}\text { Diciembre } \\
(\mathrm{n}=\mathbf{1 2 . 0 9 9 . 7 9 0})\end{array}$} \\
\hline & $\mathbf{n}$ & $\%$ & $\mathrm{n}$ & $\%$ \\
\hline \multicolumn{5}{|l|}{ Sexo } \\
\hline Hombres & 4.423 .237 & 37,2 & 4.626 .922 & 38,2 \\
\hline Mujeres & 7.471 .086 & 62,8 & 7.472 .869 & 61,8 \\
\hline \multicolumn{5}{|l|}{ Edad } \\
\hline 10 a 24 años & 620.253 & 5,2 & 515.826 & 4,3 \\
\hline 25 a 54 años & 6.947 .308 & 58,4 & 7.226 .722 & 59,7 \\
\hline 55 años o más & 4.326 .762 & 36,4 & 4.357 .242 & 36,0 \\
\hline \multicolumn{5}{|l|}{ Nivel educativo } \\
\hline Ninguna & 1.476 .638 & 12,4 & 1.436 .340 & 11,9 \\
\hline Primaria y secundaria & 3.429 .825 & 28,8 & 3.360 .542 & 27,8 \\
\hline Media & 3.740 .983 & 31,5 & 4.139 .439 & 34,2 \\
\hline Técnica & 1.472 .525 & 12,4 & 1.294 .235 & 10,7 \\
\hline Profesional y posgrado & 1.774 .351 & 14,9 & 1.859 .065 & 15,4 \\
\hline \multicolumn{5}{|l|}{ Tamaño del hogar } \\
\hline Unipersonal & 1.305 .914 & 11,0 & 1.313 .022 & 10,9 \\
\hline Dos personas & 2.167 .986 & 18,2 & 2.392 .293 & 19,8 \\
\hline Tres personas & 3.014 .004 & 25,3 & 3.005 .692 & 24,8 \\
\hline 4 o más & 5.406 .419 & 45,5 & 5.388 .783 & 44,5 \\
\hline \multicolumn{5}{|l|}{ Pobreza monetaria } \\
\hline Total & $12.015 .589^{\pi}$ & & & \\
\hline No pobre & 9.338 .857 & 77,7 & 9.492 .102 & 78,4 \\
\hline Pobre & 2.676 .732 & 22,3 & 2.607 .688 & 21,6 \\
\hline
\end{tabular}


El desinterés en recibir la vacuna contra el coronavirus en población de 23 ciudades colombianas, julio a diciembre de 2020

\begin{tabular}{|c|c|c|c|c|}
\hline \multirow[t]{2}{*}{ Ciudad } & \multicolumn{2}{|c|}{$\begin{array}{c}\text { Julio } \\
\left(\mathrm{n}=\mathbf{1 2 . 0 2 8 . 2 2 7 ^ { * } )}\right.\end{array}$} & \multicolumn{2}{|c|}{$\begin{array}{c}\text { Diciembre } \\
\left(n=\mathbf{1 2 . 0 6 4 . 7 0 0}^{* *}\right)\end{array}$} \\
\hline & $\mathbf{n}$ & $\%$ & $\mathrm{n}$ & $\%$ \\
\hline Armenia & 148.678 & 1,2 & 149.012 & 1,2 \\
\hline Barranquilla AM & 743.311 & 6,2 & 762.107 & 6,3 \\
\hline Bogotá D.C. & 4.157 .217 & 34,6 & 4.219 .600 & 35,0 \\
\hline Bucaramanga AM & 523.737 & 4,4 & 511.774 & 4,2 \\
\hline Cali AM & 1.270 .455 & 10,6 & 1.245 .942 & 10,3 \\
\hline Cartagena & 426.196 & 3,5 & 413.509 & 3,4 \\
\hline Cúcuta AM & 377.157 & 3,1 & 383.092 & 3,2 \\
\hline Florencia & 82.533 & 0,7 & 80.881 & 0,7 \\
\hline Ibagué & 276.857 & 2,3 & 263.211 & 2,2 \\
\hline Manizales AM & 215.886 & 1,8 & 214.351 & 1,8 \\
\hline Medellín AM & 1.871 .818 & 15,6 & 1.891 .368 & 15,7 \\
\hline Montería & 153.061 & 1,3 & 150.272 & 1,2 \\
\hline Neiva & 161.205 & 1,3 & 163.573 & 1,4 \\
\hline Pasto & 182.929 & 1,5 & 183.774 & 1,5 \\
\hline Pereira AM & 314.727 & 2,6 & 315.295 & 2,6 \\
\hline Popayán & 123.851 & 1,0 & 121.336 & 1,0 \\
\hline Quibdó & 42.163 & 0,4 & 43.711 & 0,4 \\
\hline Riohacha & 103.795 & 0,9 & 104.445 & 0,9 \\
\hline Santa Marta & 211.124 & 1,8 & 205.628 & 1,7 \\
\hline Sincelejo & 116.935 & 1,0 & 115.951 & 1,0 \\
\hline Tunja & 97.265 & 0,8 & 99.226 & 0,8 \\
\hline Valledupar & 180.589 & 1,5 & 179.732 & 1,5 \\
\hline Villavicencio & 246.739 & 2,1 & 246.911 & 2,0 \\
\hline
\end{tabular}

Fuente: elaboración propia con base en la Encuesta Pulso Social, 2020, DANE.

El no tener interés en recibir la vacuna difirió por sexo, grupo de edad, nivel educativo y tamaño del hogar y por cada una de esas características mostró un aumento entre julio y diciembre. De igual manera se diferenció por pobreza monetaria y ciudad, características que también evidenciaron incremento entre septiembre y diciembre (tabla 3). La no disposición a recibir la vacuna fue mayor en las mujeres. Tanto en hombres como en mujeres, la falta de interés aumentó 12 y 13 puntos porcentuales respectivamente, entre julio y diciembre, asimismo aumentó en todos los grupos de edad y fue más alta en el grupo de 55 años y más entre julio y diciembre respecto de los demás grupos de edad y con un aumento entre meses de 12 puntos porcentuales. Igualmente, el desinterés fue mayor entre las personas con baja escolaridad y entre julio y diciembre aumentó en todas las categorías del nivel educativo: en las personas con niveles de primaria y secundaria, aumentó 14,36 puntos porcentuales. Por tamaño de hogar, la ausencia de interés fue variable, mientras en julio fue más alta en los unipersonales, en diciembre lo fue para los conformados por dos personas, y el mayor incremento entre meses, 16,5 puntos porcentuales, correspondió a los hogares conformados por tres personas. La ausencia de interés fue relativamente más alta en el grupo de pobres. En las 23 ciudades la no intención de recibir la vacuna aumentó entre septiembre y diciembre en 10,6 puntos porcentuales y Cali presentó los valores más altos en septiembre $(56,1 \%)$ y diciembre $(46,5 \%)$, con un aumentó entre meses de 10,4 puntos porcentuales; sin embargo, el incremento más alto entre meses fue para Santa Marta con 19,5 puntos porcentuales. 
Pablo Enrique Chaparro Narváez / Carlos Andrés Castañeda Orjuela

Tabla 3 Prevalencia del desinterés en recibir la vacuna contra el coronavirus, Colombia, julio - diciembre 2020

\begin{tabular}{|c|c|c|c|c|c|}
\hline \multirow[b]{3}{*}{ Total } & \multirow{3}{*}{\begin{tabular}{|c|c}
$\begin{array}{c}\text { Julio } \\
(\mathrm{n}=3.257 .667)\end{array}$ \\
27,39 \\
\end{tabular}} & \multirow{3}{*}{$\begin{array}{c}\begin{array}{c}\text { Diciembre } \\
(\mathbf{n}=4.855 .047)\end{array} \\
40,13\end{array}$} & \multicolumn{3}{|c|}{$\begin{array}{c}\text { Diferencia entre las estimaciones } \\
\text { de diciembre y julio }\end{array}$} \\
\hline & & & \multirow{2}{*}{$\begin{array}{c}\text { Diferencia } \\
12,74\end{array}$} & \multicolumn{2}{|c|}{ IC $95 \%$} \\
\hline & & & & 12,69 & 12,77 \\
\hline \multicolumn{6}{|l|}{ Sexo } \\
\hline Hombres & 23,81 & 35,91 & 12,10 & 12,04 & 12,16 \\
\hline Mujeres & 29,51 & 42,74 & 13,23 & 13,18 & 13,28 \\
\hline \multicolumn{6}{|l|}{ Edad } \\
\hline 10 a 24 aก̃os & 20,89 & 41,80 & 20,92 & 20,75 & 21,09 \\
\hline 25 a 54 años & 25,23 & 37,78 & 12,55 & 12,50 & 12,60 \\
\hline 55 años o más & 31,78 & 43,82 & 12,03 & 11,97 & 12,10 \\
\hline \multicolumn{6}{|l|}{ Nivel educativo } \\
\hline Ninguna & 35,88 & 47,73 & 11,85 & 11,74 & 11,96 \\
\hline Primaria y secundaria & 30,35 & 44,78 & 14,44 & 14,36 & 14,51 \\
\hline Media & 27,19 & 40,56 & 13,37 & 13,31 & 13,44 \\
\hline Técnica & 21,05 & 32,25 & 11,20 & 11,10 & 11,31 \\
\hline Profesional y posgrado & 20,29 & 30,15 & 9,86 & 9,77 & 9,95 \\
\hline \multicolumn{6}{|l|}{ Tamaño del hogar } \\
\hline Unipersonal & 36,66 & 41,78 & 5,12 & 5,00 & 5,23 \\
\hline Dos personas & 28,22 & 43,76 & 15,54 & 15,46 & 15,63 \\
\hline Tres personas & 25,18 & 41,70 & 16,52 & 16,44 & 16,59 \\
\hline 4 o más & 26,05 & 37,23 & 11,18 & 11,13 & 11,24 \\
\hline \multicolumn{6}{|l|}{ Pobreza monetaria } \\
\hline Total & $4.503 .831^{9}$ & & & & \\
\hline No pobre & 36,98 & 39,74 & 2,76 & 2,71 & 2,80 \\
\hline Pobre & 39,24 & 41,53 & 2,30 & 2,21 & 2,38 \\
\hline
\end{tabular}

"Inició recolección de datos a partir de septiembre.

Los IC 95\% de las diferencias que excluyen el cero son estadísticamente significativos,

\begin{tabular}{|l|c|r|r|r|r|}
\hline \multicolumn{1}{|c|}{ Ciudad } & $\begin{array}{c}\text { Julio } \\
\text { (n=3.257.667) }\end{array}$ & $\begin{array}{c}\text { Diciembre } \\
(\mathbf{n = 5 . 1 0 1 . 8 4 9 )}\end{array}$ & \multicolumn{3}{|c}{$\begin{array}{c}\text { Diferencia entre las estimaciones de } \\
\text { diciembre y julio }\end{array}$} \\
\hline Total & 31,66 & 42,29 & 10,62 & 10,58 & 10,66 \\
\hline Armenia & 34,69 & 43,04 & 8,35 & 8,00 & 8,70 \\
\hline Barranquilla AM & 32,74 & 37,39 & 4,65 & 4,49 & 4,80 \\
\hline Bogotá D.C. & 28,15 & 41,11 & 12,96 & 12,89 & 13,02 \\
\hline Bucaramanga AM & 37,93 & 51,22 & 13,29 & 13,10 & 13,48 \\
\hline Cali AM & 48,93 & 59,49 & 10,56 & 10,44 & 10,69 \\
\hline Cartagena & 25,81 & 42,97 & 17,16 & 16,96 & 17,36 \\
\hline Cúcuta AM & 36,39 & 45,23 & 8,85 & 8,63 & 9,07 \\
\hline Florencia & 27,76 & 43,92 & 16,16 & 15,70 & 16,62 \\
\hline Ibagué & 35,16 & 50,30 & 15,14 & 14,88 & 15,40 \\
\hline Manizales AM & 25,79 & 35,34 & 9,55 & 9,27 & 9,82 \\
\hline Medellín AM & 28,83 & 34,13 & 5,30 & 5,21 & 5,39 \\
\hline Montería & 25,53 & 39,39 & 13,86 & 13,53 & 14,19 \\
\hline Neiva & 32,98 & 43,00 & 10,02 & 9,69 & 10,35 \\
\hline Pasto & 24,71 & 30,86 & 6,15 & 5,86 & 6,44 \\
\hline Pereira AM & 37,89 & 46,19 & 8,30 & 8,06 & 8,54 \\
\hline Popayán & 32,21 & 38,87 & 6,66 & 6,28 & 7,04 \\
\hline Quibdón & 20,19 & 28,99 & 8,79 & 8,22 & 9,37 \\
\hline Riohacha & 19,32 & 32,51 & 13,19 & 12,81 & 13,56 \\
\hline Santa Marta & 19,35 & 39,19 & 19,84 & 19,57 & 20,11 \\
\hline Sincelcjo & 37,39 & 42,13 & 4,75 & 4,35 & 5,14 \\
\hline Tunja & 24,13 & 34,47 & 10,34 & 9,95 & 10,74 \\
\hline Valledupar & 28,24 & 38,51 & 10,27 & 9,96 & 10,58 \\
\hline Villavicencio & 30,83 & 46,98 & 16,16 & 15,89 & 16,43 \\
\hline & & & & & \\
\hline
\end{tabular}

Fuente: elaboración propia con base en la Encuesta Pulso Social, 2020, DANE.

El desinterés en recibir la vacuna tuvo un comportamiento creciente durante la mayor parte del periodo de estudio. El descenso se presentó a partir de noviembre por sexo, edad de 25 a 54 años, para quienes tenían estudios de primaria y secundaria y media, para hogares con dos o más personas, y para personas pobres y no pobres. En el grupo de edad de 55 y más años 
se mantuvo el aumento durante todo el periodo. Las 23 ciudades tuvieron entre septiembre y diciembre crecimiento en el desinterés en recibir la vacuna, Cali fue la ciudad con los valores más altos y Santa Marta la que mostró el mayor incremento. Ver la figura 1.

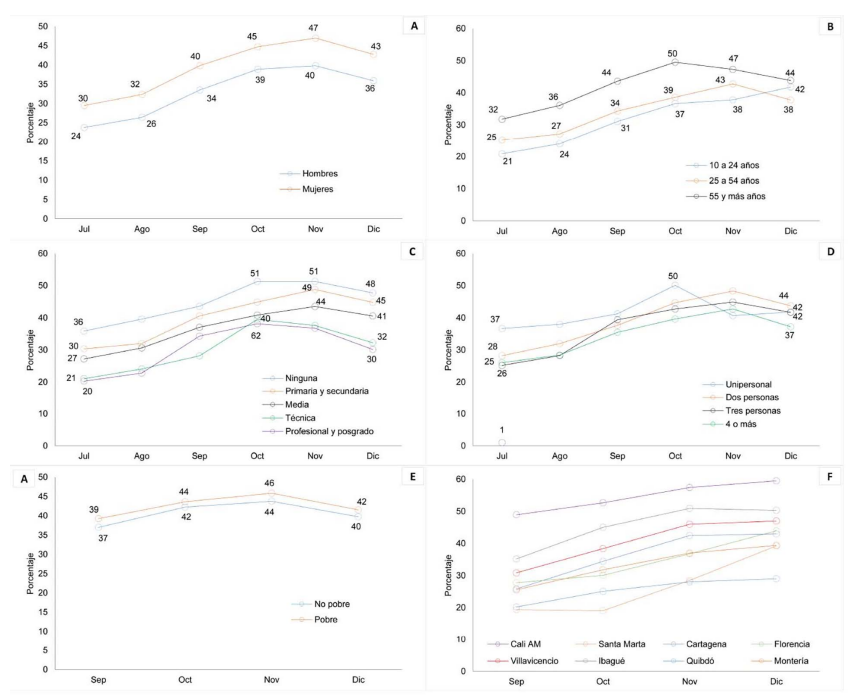

Figura 1 Comportamiento del desinterés en recibir la vacuna

contra el coronavirus, Colombia, julio - diciembre 2020

Fuente: elaboración propia con base en la Encuesta Pulso Social, 2020, DANE.

Las razones mencionadas con mayor frecuencia por las personas que no tenían interés en recibir la vacuna y que respondieron la encuesta de diciembre, se relacionaban con la idea de que la vacuna podría ser insegura debido a los potenciales efectos adversos $(65,5 \%)$ y la no creencia en que la vacuna pueda ser lo suficientemente efectiva $(16,4 \%)$. Ver la tabla 4. En 9,3 puntos porcentuales aumentó entre noviembre y diciembre la creencia que la vacuna podía ser insegura debido a los potenciales efectos adversos (65,5\% versus $56,2 \%)$, y en 5,4 puntos porcentuales se redujo la creencia que la vacuna se utilizaría para manipularlo (3,8\% versus $9,7 \%)$. 
Tabla 4 Principales razones para no tener interés en recibir la vacuna contra el coronavirus, Colombia, noviembre y diciembre 2020

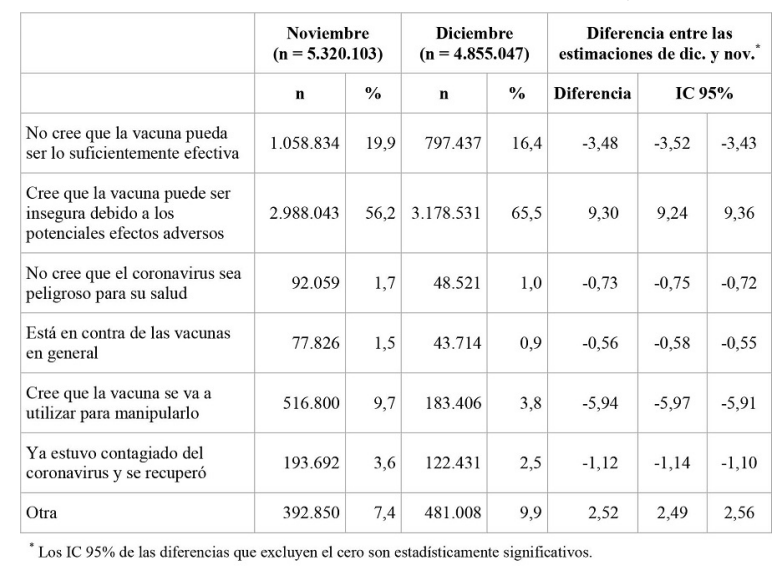

Fuente: elaboración propia con base en la Encuesta Pulso Social, 2020, DANE.

\section{Discusión}

La falta de interés en recibir la vacuna contra el coronavirus en 23 ciudades colombianas aumentó de $27 \%$ en julio a $40 \%$ en diciembre de 2020 . Además, se identificaron variaciones demográficas y geográficas en las prevalencias de no aceptación. La no aceptación puede dificultar la obtención de la inmunidad colectiva contra el COVID-19.

Este estudio indicó que la prevalencia fue mayor en mujeres, en los grupos de 55 y más años, sin ningún nivel de escolaridad, en hogares conformados por dos personas o menos, en los grupos pobres y, principalmente, en ciudades como Cali y Bucaramanga y sus áreas metropolitanas. Las diferencias en las estimaciones efectuadas entre diciembre y julio señalaron que la falta de interés por recibir la vacuna tuvo un mayor aumento en el grupo de edad de 10 a 24 años y en las ciudades de Santa Marta y Cartagena. En general, el comportamiento del desinterés en recibir la vacuna fue ascendente y a partir de octubre comenzó a descender excepto para el grupo de edad de 10 a 24 años y en las 23 ciudades. La principal razón para no tener interés en recibir la vacuna que más incremento experimentó fue creer que la vacuna puede ser insegura debido a los potenciales efectos adversos.

El 27\% de los encuestados en julio no tenía la intención de recibir la vacuna. En ese periodo la población sobrellevó el aislamiento y el distanciamiento social como principales medidas de prevención. A diciembre, los niveles de intención cambiaron significativamente pasando a $40 \%$. El mayor cambio en la intención se produjo en el grupo de edad de 20 a 24 años, que incrementó en 20 puntos porcentuales. En diciembre, mes de la reactivación económica, la población se dedicó a hacer compras y preparativos para las celebraciones decembrinas y fue evidente la relajación en la aplicación de las medidas recomendadas para evitar el contagio, lo que se relacionó con un segundo pico entre enero y febrero de 2021 (31). 
A pesar de la disponibilidad de servicios de vacunación, el retraso en la aceptación o el rechazo de la vacunación es bien conocida para otros tipos de vacunas y ha venido en aumento en los últimos años (5). De acuerdo con los resultados presentados, esta situación fue vivida en 23 ciudades colombianas en relación con la vacuna contra el coronavirus y es reflejo del panorama observado en otras regiones del mundo. De hecho, los niveles de desinterés en recibir la vacuna encontrados fueron mayores a los reportados en Estados Unidos (20\%) (15), Francia (24\%) (11) y Europa $(7,2 \%$ a $26 \%)(12,14)$.

Las diferencias en la falta de interés por recibir la vacuna se evidenciaron por sexo y por grupos de edad como se reportó para algunos países europeos (12). Los hombres tuvieron menor desinterés que las mujeres por recibir la vacuna. Se percibe que los hombres tienden a ser más receptivos a las vacunas COVID-19 y son más propensos a aceptar la vacunación, mientras que en las mujeres resulta más probable que se preocupen por contraer la infección, por los efectos secundarios y por la toma de medidas de protección como el uso de mascarillas, el lavado de manos y el distanciamiento social (32). En relación con la edad se reportó que los hombres que no estaban dispuestos a vacunarse eran los más jóvenes de 18 a 24 años (12). Con base en estos resultados, los esfuerzos preventivos y de educación y comunicación podrían estar dirigidos a las mujeres y a las personas menores de 24 años y, en general, hacia aquellos en los que se evidenció un menor interés.

La falta de interés por recibir la vacuna difiere según el nivel educativo y el nivel de pobreza. Este estudio encontró que a medida que aumenta la escolaridad, se reduce el desinterés en la vacuna; aun así, el porcentaje de personas de mayor nivel educativo que tuvieron falta de interés en julio y diciembre fue de $20,3 \%$ y $30,2 \%$, respectivamente. Los estudios han reportado que la escolaridad con frecuencia se relaciona con la educación en salud, aspecto que se debe considerar al momento de establecer actividades educativas dirigidas a la población y que traten sobre la importancia de las vacunas en la reducción de la morbilidad y mortalidad debida a la COVID-19 (33). Respecto de la pobreza monetaria, el desinterés en recibir la vacuna fue más alto en el grupo de pobres, similar a los resultados de un estudio en el Reino Unido e Irlanda (34). Además, se ha observado que las personas pobres generalmente están más expuestas a enfermedades infecciosas (35). En condiciones habituales y con un sector económico sólido, a medida que aumenta el nivel educativo disminuye el indicador de desempleo y aumentan los ingresos (36), y es probable que se reduzca la exposición a las enfermedades contagiosas.

Sobre las razones para no tener interés en recibir la vacuna, dos terceras partes de los encuestados cree que la vacuna puede ser insegura debido a los potenciales efectos adversos y menos de una quinta parte no cree que la vacuna pueda ser lo suficientemente efectiva. Estos hallazgos concuerdan con los reportados en la literatura sobre los motivos frecuentes del retraso en la aceptación o el rechazo de la vacunación, a pesar de la disponibilidad de servicios de vacunación (37). Estas razones también aparecieron entre los encuestados en un estudio ruso (14) en el que los participantes afirmaron que no recibirían la vacuna, siendo las principales razones la falta de información adecuada y la confianza hacia la ciencia y la vacunación entre el público en 
general, y los esfuerzos necesarios a realizar por parte de las autoridades en salud para lograr coberturas óptimas.

Múltiples explicaciones podrían dar respuesta a la falta de interés. Entre otros aspectos se consideran las creencias políticas y las actitudes hacia las vacunas (38), la gravedad percibida de la enfermedad, el riesgo de infección y la seguridad, la eficacia, la efectividad y la necesidad de la vacuna $(32,39)$, la proliferación de teorías de conspiración en torno al coronavirus y las vacunas combinada con los movimientos antivacunas (32), la desconfianza en la medicina, la ciencia y la salud pública (40) y las disparidades existentes en la atención de la salud que surgen de condiciones sociales y económicas adversas (41).

Los esfuerzos que se realicen en torno a la prevención del COVID-19 y el optimismo por la disponibilidad de una nueva vacuna (aunque con demoras en su entrega) serán infructuosos si en la toma de decisiones se omite de los planes de vacunación, que una parte importante de la población puede negarse a participar en el programa de inmunización. El objetivo de todo programa de este tipo es alcanzar una proporción de individuos inmunes para que no sufran las consecuencias de la infección y para que toda la población termine indirectamente protegida a través de la inmunidad colectiva. Teniendo en cuenta que ese umbral de inmunidad para el SARS-CoV-2 es cercano al 67\%, se espera que una vez alcanzado, la probabilidad de que individuos aún susceptibles se encuentren con un portador infeccioso, disminuya significativamente (14). Sin embargo, se debe recordar que, en la ciudad brasileña de Manaos, con más del $70 \%$ de la población infectada siete meses después de que el virus llegara por primera vez, parece que no se había desarrollado la inmunidad colectiva (42). Todas las razones expuestas enfatizan en la falta de información adecuada y la falta de confianza hacia la ciencia y la vacunación entre el público en general (14). Por lo tanto, es necesario insistir en que los tomadores de decisiones deben considerar estrategias de información, educación y comunicación que mejoren la percepción de las vacunas por parte de la población y combatan la desinformación y noticias falsas (situación identificada como "infodemia"). La confianza pública en los programas de vacunación depende del trabajo que ellos hacen por la comunidad en lo social, político y moral, así como en lo biológico (43). Por lo tanto, la estrategia de intervención de la COVID-19 no solo debe basarse en entregar vacunas, sino también, entre otras cosas, en generar confianza en la población en sus sistemas de salud pública para enfrentar esta y futuras emergencias.

Se reconocen como limitaciones de esta investigación, primero, un posible sesgo de selección debido a que los participantes debían tener acceso a un teléfono para participar, hecho que pudo haber excluido a personas pobres y mayores que hacen parte de grupos vulnerables al COVID-19 y que, como consecuencia, haya llevado a una subestimación de los que no tenían interés en la vacuna. Segundo, al ser un estudio basado en encuestas con datos generados en línea no se pudo verificar la veracidad de las respuestas de los participantes. Tercero, las encuestas son tomadas en determinados momentos y las creencias y actitudes generales hacia la vacunación 
pueden estar impulsadas por la intención de vacunación en ese momento (44). Cuarto, las distintas rondas de la encuesta se realizaron en un contexto dinámico y cambiante, sujeto a variaciones debidas a la percepción del peligro que entraña la enfermedad y el desarrollo de la vacuna (13) que pudieron influir en la intención de vacunarse, y los resultados pueden no reflejar las razones actuales para no tener la intención de recibir la vacuna. Quinto, no se tuvo información sobre las razones para no tener interés en recibir la vacuna de la categoría "otra". Sexto, aunque se identificó el comportamiento del desinterés en recibir la vacuna, no fue posible realizar un análisis longitudinal debido a que la muestra no configuró una cohorte. Por último, es posible que los resultados no sean comparables con otras encuestas debido a las diferencias en la muestra considerada, así como a las preguntas relacionadas con la intención de la vacunación y la técnica empleada para la recolección de la información. Entre las fortalezas del estudio están el haber considerado una encuesta con muestra probabilística, la estratificación por las variables de persona y lugar para identificar la no aceptación de la vacuna COVID-19 y la recopilación de información en distintos momentos en el tiempo para examinar la intención de vacunación.

Para concluir, se encontró que la falta de interés por recibir la vacuna contra el coronavirus en 23 ciudades colombianas aumentó entre julio y diciembre de 2020. Conocer sobre la falta de interés por la vacuna en la época en que se inicia la vacunación puede ayudar a las autoridades de salud a elaborar políticas relacionadas con estrategias educativas y de comunicación destinadas a aumentar la confianza en la eficacia y seguridad de la vacuna, para que de esa manera se contribuya con la ruptura de la cadena de transmisión $(4,5)$. El reto es alcanzar el umbral de inmunidad, debido a que una proporción considerable de la población que le falta interés en recibir la vacuna puede propagar el virus y contraer la enfermedad, incluso después de que se haya logrado la inmunidad colectiva (12), a pesar de la incertidumbre sobre cuánto tiempo puede durar la protección. Las estrategias deben considerar el nivel educativo de las poblaciones y abordar los problemas que generan desconfianza.

\section{Consideraciones éticas}

Este estudio utilizó información de fuente secundaria anonimizada y agrupada, por lo que no requirió de aval ético. Además, la información es de interés en salud pública.

\section{Contribución de los autores}

Los dos autores participaron en el diseño del estudio, el análisis estadístico, la discusión de resultados y la redacción del manuscrito.

\section{Financiación}

Este trabajo fue realizado con recursos de funcionamiento del Observatorio Nacional de Salud del Instituto Nacional de Salud de Colombia.

\section{Conflictos de interés}

Los autores declaran que no existe conflicto de interés. 


\section{Referencias}

1. Johns Hopkins University. COVID-19 Dashboard by the Center for Systems Science and Engineering (CSSE) at Johns Hopkins University (JHU). 2021. https://coronavirus.jhu.edu/map.html

2. Gao Q, Bao L, Mao H, Wang L, Xu K, Yang M, et al. Development of an inactivated vaccine for SARSCoV-2. Science (80). 2020;81(July):77-81. https://doi.org/10.1126/science.abc1932

3. Nuño M, Chowell G, Gumel AB. Assessing the role of basic control measures, antivirals and vaccine in curtailing pandemic influenza: Scenarios for the US, UK and the Netherlands. J R Soc Interface. 2007;4(14):505-521. https://doi.org/10.1098/rsif.2006.0186

4. Malik AA, McFadden SAM, Elharake J, Omer SB. Determinants of COVID-19 vaccine acceptance in the US. EClinicalMedicine. 2020;26:100495. https://doi.org/10.1016/j.eclinm.2020.100495

5. Thunstrom L, Madison A, Finnoff D, Stephe N. Hesitancy towards a COVID-19 vaccine and prospects for herd immunity. SSRN Electron J. 2020.

6. Silverman E. STAT-Harris Poll: The share of Americans interested in getting COVID-19 vaccine as soon as possible is dropping. STAT; 2020. https://www.statnews.com/pharmalot/2020/10/19/covid19-cor onavirus-pandemic-vaccine-racial-disparities/

7. Reinhart R. More Americans Now Willing to Get COVID-19 Vaccine. Gallup; 2020. https://news.gallu p.com/poll/325208/americans-willing-covid-vaccine.aspx

8. Talev M. Percent who say they are likely to get the first generation COVID-19 vaccine as soon as it is available. Axios. 2020. https://www.axios.com/axios-ipsos-poll-coronavirus-index-vaccine-doubts-e9205f29-8c18-4 980-b920-a25b81 eebd84.html?mkt_tok=eyJpIjoiTm1 Wak56QTBaVGxrTmpGaSIsInQiOiJ3WXds cTJ2NmVGOEpmeUpZdXdXOElZRnR1Umptd3ZkUEpCRzRNUWdYbm5Wa29pOVdMNkxrAll rightsreserved.Noreuse

9. Hamel L, Kirzinger A, Muñana C, Brodie M. KFF COVID-19 Vaccine Monitor: December 2020. KFF. 2020. https://www.kff.org/coronavirus-covid-19/report/kff-covid-19-vaccine-monitor-decemb er-2020/

10. Peretti-Watel P, Seror V, Cortaredona S, Launay O, Raude J, Verger P, et al. A future vaccination campaign against COVID-19 at risk of vaccine hesitancy and politicisation. Lancet Infect Dis. 2020;20(7):769-770. https://doi.org/10.1016/S1473-3099(20)30426-6

11. Ward JK, Alleaume C, Peretti-watel P. The French public's attitudes to a future COVID-19 vaccine: The politicization of a public health issue. Soc Sci Med J. 2020;265:113414. https://doi.org/10.101 6/j.socscimed.2020.113414

12. Neumann-Böhme S, Varghese NE, Sabat I, Barros PP, Brouwer W, van Exel J, et al. Once we have it, will we use it? A European survey on willingness to be vaccinated against COVID-19. Eur J Heal Econ. 2020;21(7):977-982. https://doi.org/10.1007/s10198-020-01208-6 
El desinterés en recibir la vacuna contra el coronavirus en población de 23 ciudades colombianas, julio a diciembre de 2020

13. Lazarus J V., Ratzan SC, Palayew A, Gostin LO, Larson HJ, Rabin K, et al. A global survey of potential acceptance of a COVID-19 vaccine. Nat Med. 2020;27(February):225-228. http://dx.doi.org/10.10 38/s41591-020-1124-9

14. Marcec R, Majta M, Likic R. Will vaccination refusal prolong the war on SARS-CoV-2? Postgrad Med J. 2020;97(August):143-149. https://doi.org/10.1136/postgradmedj-2020-138903

15. Economía Digital. Crece el escepticismo sobre la vacuna: un $47 \%$ de españoles no quiere ponérsela. Economía Digital. 2020 Nov 18. https://www.economiadigital.es/politica/crece-escepticismo-la-vac una-47-espanoles-no-quiere-ponersela_20107279_102.html

16. Organización Mundial de la Salud. Diez cuestiones de salud que la OMS abordará este año. 2021. http s://www.who.int/es/news-room/spotlight/ten-threats-to-global-health-in-2019

17. MacDonald NE, SAGE Working Group on Vaccine Hesitancy. Vaccine hesitancy: Definition, scope and determinants. Vaccine. 2015;33:4161-4164. https://doi.org/10.1016/j.vaccine.2015.04.036

18. Bednarczyk RA. Commentary examining the "why" of vaccine hesitancy. Heal Psychol. 2018;37(4):316-317. https://doi.org/10.1037/hea0000596

19. Hornsey MJ, Harris EA, Fielding KS. The Psychological Roots of Anti-Vaccination Attitudes: A 24Nation Investigation. Heal Psychol. 2018;37(4):307-315. https://doi.org/10.1037/hea0000586

20. Pelullo CP, Della Polla G, Napolitano F, Di Giuseppe G, Angelillo IF. Healthcare workers' knowledge, attitudes, and practices about vaccinations: A cross-sectional study in Italy. Vaccines. 2020;8(2). ht tps://doi.org/10.3390/vaccines 8020148

21. Samoff E, Dunn A, Vandevanter N, Blank S, Weisfuse IB. Predictors of acceptance of hepatitis B vaccination in an urban sexually transmitted diseases clinic. Sex Transm Dis. 2004;31(7):415-420. https://doi.org/10.1097/01.olq.0000130533.53987.78

22. Brewer NT, Hallman WK. Subjective and objective risk as predictors of influenza vaccination during the vaccine shortage of 2004-2005. Clin Infect Dis. 2006;43(11):1379-1386. https://doi.org/10.108 $6 / 508466$

23. Burgess DC, Burgess MA, Leask J. The MMR vaccination and autism controversy in United Kingdom 1998-2005: Inevitable community outrage or a failure of risk communication? Vaccine. 2006;24(18):3921-3928. https://doi.org/10.1016/j.vaccine.2006.02.033

24. Petts J, Niemeyer S. Health risk communication and amplification: Learning from the MMR vaccination controversy. Heal Risk Soc. 2004;6(1):7-23. https://doi.org/10.1080/13698570410001678284

25. Quinn SC, Thomas T, Kumar S. The anthrax vaccine and research: Reactions from postal workers and public health professionals. Biosecurity and Bioterrorism. 2008;6(4):321-333. https://doi.org/10.10 89/bsp.2007.0064

26. Zitner T. Vaccine hesitancy and the unique challenge of COVID-19. Washington, DC; 2020. https://journals.lww.com/ambulatorycaremanagement/Fulltext/2021/01000/COVID_19_and_ Vaccine_Hesitancy_A_Challenge_the.10.aspx

27. Silveira MM, Moreira GMSG, Mendonça M. DNA vaccines against COVID-19: Perspectives and challenges. Life Sci. 2021;267(September 2020):118919. https://doi.org/10.1016/j.lfs.2020.118919 
28. DANE. Encuesta Pulso Social. 2020 [cited 2021 Jan 20]. https://www.dane.gov.co/index.php/estadist icas-por-tema/encuesta-pulso-social

29. DANE. Metodologia Gran Encuesta Integrada de Hogares. Colección Documentos - Actualización 2009. Bogotá D.C.; 2009.https://www.dane.gov.co/files/investigaciones/fichas/Gran_encuesta_integrada_ hogares.pdf

30. DANE. Metodología General Gran Encuesta Integrada de Hogares. Bogotá D.C.; 2016. https://www.d ane.gov.co/files/investigaciones/fichas/empleo/metodologia_GEIH-01_V9.pdf

31. Instituto Nacional de Salud. Observatorio Nacional de Salud. Módulo de datos COVID - 2019 en Colombia. 2021. https://www.ins.gov.co/Direcciones/ONS/modelos-covid-19

32. Lin C, Tu P, Beitsch LM. Confidence and receptivity for covid\#19 vaccines: A rapid systematic review. Vaccines. 2021;9:16. https://doi.org/10.3390/vaccines9010016

33. Head KJ, Kasting ML, Sturm LA, Hartsock JA, Zimet GD. A National Survey Assessing SARS-CoV-2 Vaccination Intentions: Implications for Future Public Health Communication Efforts. Sci Commun. 2020;42(5):698-723. https://doi.org/10.1177/1075547020960463

34. Murphy J, Vallières F, Bentall RP, Shevlin M, Mcbride O, Hartman TK et al. Psychological characteristics associated with COVID-19 vaccine hesitancy and resistance in Ireland and the United Kingdom. Nat Commun. 2021;12(29):1-15. http://dx.doi.org/10.1038/s41467-020-20226-9

35. Noordhout CM De, Devleesschauwer B, Salomon JA, Turner H, Cassini A, Colzani E et al. Disability weights for infectious diseases in four European countries: comparison between countries and across respondent characteristics. Eur J Public Health. 2017;28(1):124-133. https://doi.org/10.1093/eurpu $\mathrm{b} / \mathrm{ckx} 090$

36. United States Department of Labor. Unemployment rates and earnings by educational attainment. 2019. https://www.bls.gov/emp/chart-unemployment-earnings-education.htm

37. The Vaccine Confidence Project, London School of Hygiene \& Tropical Medicine. The state of vaccine confidence 2015. London; 2015. https://static1.squarespace.com/static/5d4d746d648a4e0001186e38 /t/5d75156b63cb4f265725de12/1567954291535/VCP_The-State-of-Vaccine-Confidence_2015.pdf

38. Kennedy J. Populist politics and vaccine hesitancy in Western Europe: an analysis of national-level data. Eur J Public Health. 2019;29(3):512-516. https://academic.oup.com/eurpub/article-abstract/29/3/51 $2 / 5364298$

39. Wang K, Lai E, Wong Y, Fai K, Wai A, Cheung L, et al. Intention of nurses to accept coronavirus disease 2019 vaccination and change of intention to accept seasonal influenza vaccination during the coronavirus disease 2019 pandemic: A cross-sectional survey. Vaccine. 2020;38:7049-7056. https:/ /doi.org/10.1016/j.vaccine.2020.09.021

40. Paul E, Steptoe A, Fancourt D. Attitudes towards vaccines and intention to vaccinate against COVID-19: Implications for public health communications. Lancet Reg Heal - Eur. 2021;1:100012. https://doi. org/10.1016/j.lanepe.2020.100012 
41. Ruiz JB, Bell RA. Predictors of intention to vaccinate against COVID-19: Results of a nationwide survey. Vaccine. 2020;39:1080-1086. https://doi.org/10.1016/j.vaccine.2021.01.010

42. Buss LF, Prete CA, Abrahim CMM, Mendrone A, Salomon T, De Almeida-Neto C, et al. Three-quarters attack rate of SARS-CoV-2 in the Brazilian Amazon during a largely unmitigated epidemic. Science (80- ). 2021;371(6526):288-292. https://doi.org/10.1126/science.abe9728

43. Harrison EA, Wu JW. Vaccine confidence in the time of COVID-19. Eur J Epidemiol. 2020;35(4):32530. https://doi.org/10.1007/s10654-020-00634-3

44. Sherman SM, Smith LE, Sim J, Amlôt R, Cutts M, Dasch H, et al. COVID-19 vaccination intention in the UK: results from the COVID-19 vaccination acceptability study (CoVAccS), a nationally representative cross-sectional survey. Hum Vaccines Immunother. 2020;00(00):1-10. https://doi.org $/ 10.1080 / 21645515.2020 .1846397$ 